\title{
Die Theorie der Herstellung und der Stabilität kolloider Lösungen, I.
}

\author{
Von P. P. von Weimarn. St. Petersburg. \\ Meinem Freunde WOLFGANG OSTWALD gewidmet. \\ Vorbericht.
}

Unter obiger Ueberschrift habe ich zwei Abhandlungen. die genau genommen in nicht sehr engem Zusammenhange sich befinden. vereinigt:

a) Die wichtigsten Grundlagen der allgemeinen Theorie der Dispersions-Methoden zur Herstellung disperser Systeme.

b) Die wichtigsten Grundlagen der allgemeinen Theorie der Kondensations-Methoden zur Herstellung disperser Systemey.

Die Vorstellungen, welche als Grund dieser Theorien gelegt sind, sind sehr einfach:

Die Kondensationsmethoden stellen sich als stark gehemmte

Prozesse der Kristallisation (für Suspensoide) und

Verflüssigung (für Emulsoide) vor;

Die wichtigsten Dispersionsmethoden (ainfache Peptisation) siellen sich als ein stark gebeinmter LösungsprozeB der dispersen Niederschläge vor.

AuBer ihrer augenscheinlichen Einfachheit besitzen diese Vorstellungen die Verneinung der Spezifität des kolloiden Zustandes, resp: die Behauptung seiner Allgemeinheit.

1) Journ. d. russ. chem. Ges. 42, 214-226; 453-474 (1910). 


\section{a) Die wichtigsten Grundlagen der allgemeinen Theorie der Dispersions-Methoden zur Herstellung disperser Systeme.")}

\section{Einleitung.}

hy meiner größeren Abhandlung „Zur Lehre von den $Z$ u-

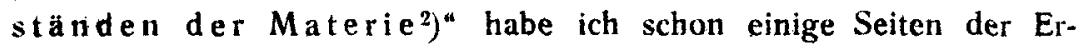
klärung der Theorie der Peptisation gewidmet, die durch den Prozeh der âberst langsamen Lösung der dispersen Phase bedingt wird ${ }^{3}$ ). Es versteht sich von selbst, daB ich damit nicht sagen wollte, daB die Peptisationserscheinungen in allen Fallen ausschlieblich durch den ProzeB der langsamen Teilchenlösung der dispersen Phase bedingt werden, denn mir waren die Peptisationserscheinungen der Kolloide durch Lōsungen hochmolekularer Körper (z. B. Gelatine, Agar-Agar u. a. m.) wohl bekannt. Namentlich im letzten Fall kann, wie es doch einem jeden einleuchtet, von einem langsamen Losen der dispersen Phase nicht die Rede sein.

Es bietet keine besonderen Schwierigkeiten, alle Methoden der Peptisation und sogar alle Dispersions-Methoden durch eine einzige Theorie zu vereinigen; auf die letztere kam ich zu Weihnachten 1908. und sie hätte in meiner Abhandlung "Zur Lehrevon den $\mathrm{Zu}$ ständen der Materie“ erscheinen müssen, was aber, wie es den Lesern schon bekannt ist ${ }^{4}$ ), dank dem veränderten Druckmodus längerer Abhandlungen nicht geschehen konnte.

In der Befürchtung, daß meine Ideen nicht richtig aufgefaßt werden könnten, sehe ich mich veranlakt, diese kurze Abhandlung, in der nur die wichtigsten Grundlagen meiner Theorie ausgetührt werden, zu schreiben ${ }^{5}$ ). Meine Befürchtungen beruhen durchaus auf einer realen Basis: So hat mein verehrter und leurer Freund Wolt -

1) Vgi. ebenfalls meine Berichte an die chem. Abt. der russ. physik.-chen. Gesellschaft: 1. Der Parallelismus $z$ wischen der Peptisation und Auflösung (4. Dez. 1908); 2. Die Peptisation der kolloiden Niederschläge von BaSO, und andrer Körper durch Agar-Agar (T. Mai 1909).

2) Koll.- Zeitschr. 4, 123-130 (1909).

8) Koll.-Zeitschr. 4, 125-126 (1909).

4) KoH. -Zeitschr. 5, 221 (1909).

5) Genaueres siche inein Werk ,Kolloidwissenschaftund Struktur der Materie“. (In Vorbereitung.) 
gang Ostwald besondere Aufmerksamkeit meinen Untersuchungen geschenkt und schreiht in seinem ausgezeichneten Werk , irundriB der Kolloidchemie" anf Seite 108, daß meine Theorie erstens kompliziert sei und zweitens die Peptisationserscheinungen grobdisperser Systeme nicht erkläre. Ferner erhielt ich vor kurzem von dem hervorragenden Dresdener Kolloidforscher A. Lottermoser ein liebenswürdiges Schreiben, in dem er unter anderem darauf hinweist, daB, obgleich unsere Ansichten über die Peptisationserscheinungen nicht wesentlich voneinander divergieren. er doch an meiner Bedingung II für alle Peptisationen zweifle.

Selbstverständlich wird, wenn die Peptisationserscheinungen nicht einfach sind, auch ihre Theorie kompliziert; was aber die Peptisation grobdisperser Niederschläge anlangt, so mub ich bemerken, dak meinc Theorie auch hierfür anwendbar ist. Was die Meinung meines hochverehrten Kollegen $A$. Lot termoser betrifft, so mub ich hinzufügen. dab es weiterhin sich zeigen wird, dak meine Bedingung II in allen Fällen der Peptisation ohne Ausnahne Cieltung hat, weun nicht rein mechanische Faktoren initwirken (wie $z$. B. Verringerung des spezifischen Gewichts der dispersen Teilchen bei der Peptisation der Kolloide durch Lösungen hochmolekularer Körper u. a. 111.).

Die weiter unten entwickelte Theorie befindet sich natürlich in mehr oder weniger naher Heziehung zu den Ansichten meiner Vorgänger auf diesem Gebiete. Un mich kurz zu fassen, werde ich dic Geschichte dieser Frage nicht erörtern, halte mich aber für verpflichtet. darauf hinzuweisen, dah von besonderem EinfluB auf meinen Gidankengang die Arbeiten von A. Lottermoser. G. Quinclu ("Umhüllungstheorie ${ }^{*}$ ), F. C. Donnan und R. Z sigmondy waren.

Die Grundidee meiner Thcorie, welche alle Dispersionsmethode $n^{1}$, zur Herstellung disperser Systeme veraltgemeinert, kann folgendermaken formuliert werden: Wenn a d der Oberflache der dispersen Teilchen aus beliebigen Gründen die Intensitat der lösenden Kräfte wächst, aber nicht die Gröbe übersteigt, bei der die Uinkristallisationsgeschwindigkeit oder die Lösungsgeschwindigkeit eine bedeutende wird, so werden die dispersen Teilchen durch das Dispersionsmittel peptisiert.

1) Die elektrische Zerstäubung der Metalle (G. Bredig-The Svedberg) und die Zerstäubung durch kurze Lichtwellen (The Svedberg, Koll.-Zeitschr. $6,129-136,1910$ ) rechne ich $z u$ den Kondensationsmethoden. 


\section{Klassifikation der Peptisationsmethoden.}

In nieiner Abhandlung ,Ucber einige Oberflächeneigen; chaften disperser kristallinischer Stoffe usw. ") "habe ich darauf hingewiesen, dab die Oberflächenschicht eines Kristalls sich $\mathrm{n}$ molckular-kinetischer Hinsicht den innern Schichten einer stark zomprimierten Flüssigkeil nähert; nehmen wir nun an, dab ein Kristallshen $K$ eines schwerlöslichen Körpers sich in einer im Verhält nis zum rristall schwach übersättigten l.ösung befindet, so ist dieses disperse rriställchen in gewisser Hinsicht mit einem System zweier begrenzt ;ich vermengender Flüssigkeiten zu vergleichen. Damit will ich sagen, laß der Kern des dispersen Kristalls lauf Fig. I schraffiert) umgeben ist von einer Oberflächenschicht $\Delta$, welche eine gesättigte Lösung des Dispersionsinittels in ter Kristallsubstanz darstellt und die sich in dynamischem Gleichgewicht mit der gesättigten Lösung der Kristallsubstanz im Dispersionsmittel von der licke $\delta=$; befindet. Man muß sich vergegenwärtigen. lat erstens die Konzentration des Dispersionsmittels in der Oberflächenschicht

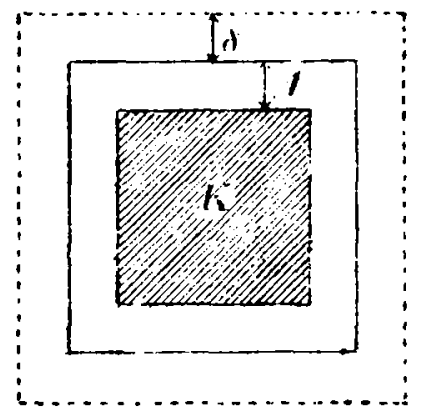

Fig. 1 mit zunehmender Entfernung von der freien Kristalloberfläche abnimum. zweitens aber bis zu einem gewissen Grade von der Konzentration der den Kristall umgebenden Lösung und von der Gegenwart der gelösten Nebensubstanzen abhängt. Die letztere Abhängig!:eit wird einem klar, wenn man in Betracht zieht, dab auf die Verletzung des Orientierungsgrades der Moleküle der Oberflächenschicht dynamische (molekular-kinetische) Prozesse hinwirkell. die sich auf der treien Kristalloberfläche abspiciell.

Aus dem Angeführten geht hervor, dat die Dichte und andere physikalische Eigenschaften des dispersen Kriställchens abhängig sind vom Dispersionsmittel und $\mathrm{Aa} B$ die Veränderung dieser Eigenschaften in einigen Fällen stark die Peptisation begünstigt. Ebenfalls geht aus dem Angeführten hervor, dab die chemische Zusammensetzung der feindispersen Phase sich von der grobdis. persen unterscheidet; mit der Veränderung der Zusam-

1) Koll.- Zeitschr. 6, $32--38$ (1910).

2) Genauer gesagt eriolgt in ter Schichtdicke $d$ eine Abnahme der Konzeiluration bis zu der Suttigungskonzentration. 
mensetzung verändern sich natürlich auch die physikalisch-chemischen Eigenschaften der dispersen Phase. Aber daraus kann man nicht die Beweise für die Richtigkeit, rein chemischer" Theorien für die Kolloide herleiten.

Stellen wir uns nun vor, dak wir eine grobe Kristallsuspension von nicht starker Konzentration irgendeines schwerlöslichen Körpers haben; führen wir in das Dispersionsmittel eine Substanz ein, die die Löslichkeit der dispersen Phase erhöht, und zwar so viel davon, dab die dispersen Teilchen eben anfangen sich langsam zu lösen, aber immer noch mit einer größeren als der Umkristallisationsgeschwindigkeit 1 ). Dann wird augenscheinlich der Dispersitätsgrad der Suspension sich langsam vergröBern und zuerst erhält man ein Sol und dann eine echte Lösung.

Unter gleichen Umständen kann man einen grobdispersen Niederschlag zuerst in den Zustand der Suspension, dann in ein Sol und schlieblich in eine echte Lösung überlïhren.

Der oben erwähnte Fall unterliegt vollkominen meiner Theorie.

Diese Beispiele der Peptisation bezeichne ich als, einfache Peptisation": gehen wir za cinem andern Fall über: Gegeben ist ein Dispersionsmittel, und darin befindet sich in Form eines dispersen Niederschlages oder als Suspension irgendein fester kristallinischer Körper; führeı wir in das Dispersionsmittel eine darin verhältnismäbig guł sich lösende (oder eine stabile kolloide Lösung ergebende) Substanz ein, die sich auch in den Oberflächenschichten der dispersen Kriställchen löst (oder an ihnen infolge kapillarer Kräfte anhaftet); und nehmen wir der Einfachheit wegen an, das die Löslichkeit der dispersen Phase durch Zusatz der angegebenen Substanz sich nicht verändert hat Man kann nun, dank der Anwesenheit auf der Oberflächenschicht der dispersen Phasen von Molekülen eines Körpers, die itn Dispersionsmittel durch die Auflösungskräfte (oder in diesem speziellen Falle durch Kapillarkräfte) festgehaiten werden, ein jedes disperses Teilchen ais ein neues $z$ us mmengesetztes disperses Teilchen ansehen; auf der Oberfläche dieses Teilchens wuchs die Inlensitäl der autlösenden Kräfte (die Festigkeit der Verbindung mit den Molekülen des Dispersionsmittels), ohne wesentlichen Einflub weder auf die Umkristallisationsgeschwindigkeit noch auf die Löslichkeit der dispersen Phase auszuüben.

1) Hier ist die Rede von der Umkristallisation, welche durch verschiedene Lőslichkeit von Teikchen nicht gleichen Dispersitätsgrades bedingt wird. 
In diesem Falle tritt eine Peptisation ein, die ich als die „zusammengesetzt $e^{\alpha}$ bezeichne.

Es kann auch rein mechanische Peptisationserscheinungen geben, wie z. B. bei der Vergrößerung der Zăhigkeit des Dispersionsmittels und a. m.; es ist auch ebenso eine Kombination zweier geschilderter Typen der Peptisation möglich, worauf ich aber hier nicht nuher eingehe.

Wie bekannt, gibt es eine "Auswaschmethode", die ebenfalls eine Peptisationsmethode darstellt: Durch Auswaschen aus der Oberflächenschicht von Molekülen, die die Festigkeit der Bindung disperser Teilchen mit dem Dispersionsmittel verringern, wird die disperse phase peptisiert.

Jetzt mőchte ich einige Beispiele für beide Arten der Peptisation anführen, wobei ich bemerke, dab meine Theorie der Dispersionsmethode am richtigsten als "Löslichkeitsbeeinflussungstheorie der Peptisation" bezeichnet werden könnte.

\section{Beispiele der einfachen Peptisation.}

In meiner Abhandlung " $Z u$ r Lehre von den $Z u s t a ̈ n d e n$ der Materie" habe ich schon auf einige Beispiele hingewiesen') und werde dieșelben nicht noch einmal wiederholen, muls aber bei zwei anschaulichen Pällen der Peptisation etwas verweilen, die ich (zusammen mit B.W. Maly s $\mathrm{h} \mathrm{hew)} \mathrm{vor} \mathrm{kurzem} \mathrm{zufällig} \mathrm{bei} \mathrm{Herstellung}$ kolloider Lösungen von $\mathrm{P}, \mathrm{Te}, \mathrm{Se}$ und $\mathrm{S}$ zu beobachten Gelegenheit hatte. Die kolloide Lösung von Tellur ${ }^{2}$ ) wurde hergestellt durch EingieBen in eine größere Menge Wasser $(1000 \mathrm{ccm})$ von einigen Kubikzentimetern einer schwachen Te-Lösung in heißèr wăsseriger $\mathrm{KOH}$-Lösung (die Konzentration der Kalilauge entsprach der Sattigung hei Zimmertemperatur). Dabei scheidet sich Tellur in freiem Zustande infolge einer Reaktion aus, welche folgendermaken ausgedrückt werden kann ${ }^{3}$ ):

$$
2 \mathrm{~K}_{2} \mathrm{Te}+\mathrm{K}_{2} \mathrm{TeO}_{3}+3 \mathrm{H}_{2} \mathrm{O} \rightrightarrows 6 \mathrm{KOH}+\mathrm{Te}_{3} .
$$

J) Koll.-Zeitschr. 4, 123-136; 5, 66 (1909).

$\Rightarrow$ ) Auf dieselbe Art habe ich schon vor langerer Zeit kolloide Losungen vieler chemischer Verbindungen erlıalten, so ergibt sich 2 . B. beim Eingieben von einigen Kubikzentimetern einer schwachen wässerigen $\mathrm{CaCrO}_{4}$ - $\mathrm{Lobsung}_{\text {in }}$ eine gröBere Menge von Aether (mit einem geringen Zusatz, von Alkohol) ein sehr stabiles Sol $\mathrm{CaCrO}_{4}$. Vgi. die Tabelle in der Koll.-Zeitscir. 4, 133 (1909).

3) Moissan, Traité de chimie minèrale T. 1,500 (1904). 
Je nach der Konzentration von Te (die Laugenmenge in der Losurig wurde konstant gehalten)' erhält man bald Sole, bald mehr oder weriger grobe Suspensionen bis zu solchen, die vollständig trübe und kaum durchscheinend waren und im Niederschlage geringe Mengen Tellur enthielten. Bei all diesen Systemen vergrößert sich allmăhlich von selbst der Dispersitätsgrad bis zur vollständigen Lösung von Te. Die Auflösung der dispersen Teilchen hängt im gegebenen Falle von der Oxydation des feinzerstäubten Tcllurs durch den Sauerstoff der Luft in Geegenwart eines Alkalis ab, wobei man, aller Wahrscheinlichkeit nach, ein leichtlösliches Kalisalz der Teliursäure erhält. Da Luft immer in Wasscr enthalten ist, so dauert der Auflösungs prozel für grobe Suspensionen vier bis sieben Tage, natïrlich wenn das Gefile hermetisch geschlossen ist und nur eine geringe Luftmenge sich über der Flüssigkeit befindet. War alut dem Boden des Gefäßes cin Te-Niederschlag. so. geniigı es häufig, denselben aufzuschütteln, um ihn zu peptisieren und auf diese Weise also am Niedersinken zu verhindern. Durch Verringerung der gelösten Luftmenge und des Alkalis kaln man natïrlich heliebig den Lösungsprozeb verlangsa:nen. Wichtig ist es zu bemerken, dabl gröhere Te-Kristalle bei Gegenwart von Saucrstoff der Luft, ron Wasser und $s c h$ wache in Alkali sich äuferst langsam oxydieren²,

Das gelöste Tellur, welches sich vorher in Zustande der Suspension odẹr eines Sois befunden, wirc' leucht durch Reduktion mit einer starken lösung von Hydrazinchlorhyirat in cin Sol von stahlblauer Farbe übergeführl. das, wie schon $\Lambda$. Gutbicr gezeigt hat, nach einigen Stunden koaguliert. Die durch dats Eingietic! von TellurLosungen crialtenen Sole sind vo' grater Farbe mit schwachem metallischen Schinmer.

Vollständig analoge Peptisationserscheinungen beohachtet man beim P. (Die Intensität der Peptisation hängt auch hier wesentlich ron der gelösten Luftmenge ab.) Kolloider Phosphor wurde hergestellt durch Eingießen von einigen Kubikzentimetern einer alkoholischen Phosphor-lösung in $1000 \mathrm{ccm}$ kaltes Wasse: $\left.{ }^{3}\right)$. Der Uehergang

1) Meine gemeinsame Arbeit mit B.W. Malyschew über Herstcllung kollo:der Lösungen von Se, TCS und $P$ wird noch im Laufe dieses Jahres in der Koll.-Zeitschr. veröffentlicht werden.

2) Siche weitere Beispi $\epsilon_{3}$ in der Koll.-Zeitschr. 4, 198-202, 315 - 317; 5, 62-68 (19719).

3) Hier will ich bemerken, daB es nir zusamnen mit B. W. Malyschesv griungen ist, ein stabiles Sol von Se von prachtvoller rótlichrosaer Farbe durch 
vom grobdispersen Niederschlag oder von grober Suspension zum Sol und Lösung dauert beim $P$ drei bis vier Tage.

Zum Schluß dieses Abschnitts möchte ich einen besonders interessanten Fall von Vergrößerung des Dispersitätsgrades anführen, bei dem die Löslicbkeitserhöhung durch Temperaturänderung erzielt wird, die Stabilität des dispersen Systems wird durch die Zähigkeit des Dispersionsmittels bedingt. Diesen Fall will ich an einem Beispiel erläutern, das ganz besonders typisch ist, obgleich mir viele zu Gebote stehen. (Die Versuche machte ich gemeinsain mit J. B. Kahan.)

Stellen wir uns zwei Lösungen von Schwefel in Aethylalkonol (ca. 99,8 Proz.) her, die eine um 0,02 Proz. herum, die andere doppeit so stark. Von der ersten Lösung giehen wir $5 \mathrm{~cm}$ in ein Probierglas und tauchen das letztere schnell in flüssige Luft, dann erhalten wir ein vollständig durchsichtiges Glas, ohne die geringsten Spuren von Opaleszenz. Nachdem ich das Probiergläschen aus der flüssigen Luft herausgenommen, tauchte ich es in absoluten Alkohol ein, um auf diese Weise die Kondensierung und das Gefrieren von Wasserdämpfen auf den Wandungen des Probiergläschens zu verhindern. In dem Verhältnis, wie die Temperatur steigt, wird auch d:e Opaleszenz des Glases stärker, bis bei weiterer Temperaturerhöhung die Opaleszenz schwächer wird, wobei liurz vor dem völligen Verschwinden das disperse Systeni eine prachtvolle violette Färbung zeigt, was auf einen äußerst hohen Dispersitätsgrad der dispersen Phase hinweist. Die zunehmende Opaleszenz entspricht dem KondensationsprozeB die abnehmende dem Dispersionsprozeb. In Anbetracht dessen, $\mathrm{da} ß$ das Dispersionsmittel bei niedriger Temperatur recht zäh ist, erhält sich die violette Opaleszenz ziemlich lange.

Nimmt man die zweite doppelt stärkere Lösung von Schwefel in Alkohol, so erhält man ein opalisierendes Glas und die übrigen Erscheinungen sind analog den oben geschilderten.

EingitBen von einigen Kubikzentimetern einer kochenden Lösung von rotem kristallinischen Se in $C_{2}$ in ein gröleres Volum (1000 ccm) kalten Aether zu erhalten. Diese Lösung koaguliert nicht beim Gefrieren in flüssiger Luft. Die einfache Herstellungsmethode, die wunderhübsche Färbung lassen diesc Herstellung des kolloiden Se als ein geeignetes Experiment für ein Kolleg erscheinen. Unter dem Einfluß des Oxydationsprozesses verändern die suspensoiden Se-Lösungen im Aether sehr langsam ihre Dispersität und gehen fast gänzlich in echte Lösungen von Verbindungen des Se niil dem Sauerstoff über. Dasselbe beobachtet man auch, aber in bedeutend geringerem MaBe bei schwachen kolloiden Lösungen von Schvefel in Wasser. 
Ich habe ein Beispiel von Erhöhung des Dispersitătsgrades dank der infolge von Temperaturveränderung eingetretenen Löslichkeitserhöhung angeführt, um zu zeigen, dab dieser Prozeb wesentlich von der einfachen Peptisation" abweicht und kein stabiles hochdisperses System ohne Einwirkung von neuen stabilisierenden Faktoren ergeben kann.

Im vorher erwähnten Beispiele der ,einfachen Peptisation" von Tellur wird die langsame Auflösung der Teilchen der dispersen Phase durch die Bildung von im Dispersionsmittel leichtlöslichem tellursauren Kali an der Oberfläche der dispersen Teilchen bedingt. Wenn wir (Fig. 2) zwei Tellurteilchen $A$ und $B$ von verschiedener Dispersität

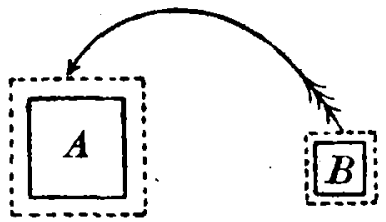

Fig. 2 haben, welche sich nah voneinander befinden, so ist es leicht $z u$ verstehen, daB A sich nicht auf Kosten vom kleineren $B$ durch Diffusion vergrobern kann. Die Oberflächen beider sind mit vou der Sattigung weit entfernten Lösungen von tellursaurem Kali umgeben, aber nicht mit einer mit Tellur gesättigten Losung von verschiedener Konzentration, weiche freies metallisches Tellur bei der Konzentrationszunahme ausscheidet, und deshalb ist es klar, daB das B-Tellurteilchen nicht durch Diffusion auf $A$ übergehen kann.

Die Vergrößerung der dispersen Teilchen auf Kosten von anderen kónte geschehen, wenn sie nicht von Alkali und von Sauerstoff umgeben wären, es befänden sich dann in der Lösung nur Moleküle von Tellur und tellursaurem Kali. Die Anwesenheit von tellursaurem Kali im Ueberschuß begünstigt aller Wahrscheinlichkeit nach die Löslichkeit von Tellur im Wasser; dabei würde natürlich die Umkristallisation von Tellur mit viel gröBerer Geschwindigkeit vor sich gehen, als wenn tellursaures Kali ohne Einfluß auf die Löslichkeit wäre. Die Umkristallisation durch Diffusion findet dann statt, wenn aus der die dispersen Teilchen umgebenden Losung metallisches Tellur (und nicht Salze der Tellursăure) sich ausscheiden; unter den angeführten Bedingungen des Experiments gibt es keine gesättigten Tellurlösungen auf der Oberfläche der dispersen Teilchen -- deshalb auch keine Umkristallisation.

Im angeführten Falle von Schwefel wird die Umkristallisation, wenn man die erhöhte Temperatur nach Erreichung eines hohen Dispersitätsgrades konstant erhält, die feinen Teilchen vernichten, denn sie werden von einer gesättigten Schwefellösung umgeben sein und 
bei erhöhter Konzentration Schwefel (und nicht Schwefelverbindungen) ausscheiden. Nur durch Verkleinerung des Diffusionskoeffizienten im Dispersionsmittel, wie z. B. durch Vergrößerung der Zähigkeit des Dispersionsmittels, wird es gelingen, das System bei hohem Dispersitătsgrade zu erhalten. Bei der Schwefellösung in Alkohol erreicht man das leicht durch schnelle Temperaturerniedrigung: bis zum Erstarren des Dispersionsmittels, wobei Inan ein feinopalisierendes Glas erhallt.

lch glaube, dab jetzt dem Leser die Bedeutung der zweiten Bedingung meiner Peptisationstheorie klar geworden ist:

II. „Die peptisierende Substanz (Peptisator) muB die Pähigkeit besitzen, bei genügend groker') Konzentration in der L.ösuug eine beständige, lösliche chemiscte Verbindung mit der Substanz, welche sich peptisiert, zu ergeben. Bei genügend kleiner Konzentration des Peptisators aher mul keine obenerwähnte Verbindung entstehen ${ }^{2}$ ).

Was die erste Bedingung der Peptisation betrifft:

I. "Die feste kristallinische Substanz nut aus Kristallchen von solch kleinen Dimensionen bestehen, bei denen die physikalischen und chemischen Eigenschaften schon Funktionen der Gribe des kristallinischen Korns sind", so ist sie an und für sich schon so klar, dats sie keiner besonderen Erläuterung bedarf, ich weise nur darauf hin, daB z. B. grohe Tellurkristalle sich mit einer Geschwindigkeit $=0$ bei crwähnter Badingung lösen und natürlich nicht peptisiert werden.

Die III. Bedingung: „Das Medium, in dem der Peptisationsprozeh vor sich geht, darf bei Abwesenheit des Peptisators in ihm die zu peptisierende Substanz praktisch gar nicht lösen $\left.{ }^{3}\right)^{\star}$, ist es sehr wichtig richtig zu verstehen.

$\mathrm{E}_{3}$ ist selbstverständlich, daB, wenn im Dispersionsmittel bei $\mathrm{Ab}$ wesenheit des Peptisators die zu peptisierende Substanz merklich sich löst, keine Peptisation erfolgt, weil entweder die ganze Substanz sich !öst, oder man eine gesättigte Lösung mit eineın Niederschlage erhält, der, auch in feindispersem Zustande hineingebracht, bei merklicher Losslichkeit in einen grobdispersen umkristallisiert. Von Bedeutung ist es, dab die Umkristallisation nicht immer stattfindet ${ }^{4}$ ). Bringen wir

1) Ich ersuche den Lcser, immer im Ange $z u$ behalten, daB große und kleine Konzentrationen nur relative Begriffe sind.

2) Koll.-Zeitschr. 4, 127 (1909,.

5) Bei sehr komplizierten Körpern braucht diese Bedingung nicht ganz eingehalirn zu werden.

9) Hier ist die Rede nur von der Diffusionsumkristallisation. Ueber "Berührungsumkristallisaiion" siehe Kolloidchem. Beihefte 1, 345 (1910). 
2. B. ein feines Kupferpulver in verdünnte Salpetersäure, so beginnen die Kupferkriställchen langsam sich zu lösen, eine Umkristallisation findet hierbei aber doch nicht statt, wenn der Lösungsprozeb auch noch so langsam vor sich geht. Die Auflösung wird hier durch Bildung von salpetersaurem Kupfer bedingt, und aus dieser Lösung kann man durch einfache Konzentrationsvergrößerung kein metallisches Kupter zurückgewinnen; es gibt in diesem Fall ebensowenig wie auch beim Tellur (siehe oben) eine Umkristallisation.

Dic Auflösung kann auch eine Folge der Bildung stabiler Komplexverbindungen sen, welche sich nur bei hohen Konzentrationen 1d. h. bei geringer Menge vom Dispersionsmittel) und niedrigen Temperaturen als Niederschlag ausscheiden. In diesem Fall und bei der gewöhnlichen Auflösung findet eine Umkristallisation statt. Wie ich schon fruiher erwähnt habe 1), hat Debray sich dieses Falles bedient, um den feinkörnigen Niederschlag $\mathrm{AgCl}$ umzukristalisieren. Es ist ersichtlich, daß es $(z . B$. hei $\mathrm{Te}, \mathrm{P}, \mathrm{Se})$ an gunstigsten ist, solche Peptisatoren zu wählen, welche eine äuberst langsame Reaktion mit der zu peptisierenden Substanz eingehen und die leichtlösliche, auch bei verhälnismäbig kleinen Konzentrationen stabile Verbindungen ergeben.

Fs ist intercssant, daß man durch dic Peptisation unter bestimmten Bedingungen grobdisperse von den feindispersen Teilchen leicht zu trennen instande ist. In der Tat werden bei der Peptisation eines Niederschlages von ungleicher Korngröß̧e die feineren Körnchen im Dispersionsmittel früher suspendiert als die gröhieren, wobei die größten auf dem Boden des Gefälbes unpeptisiert bleiben (in einem gewissen \%eitraum đer Peptisation): wenı man nun die kolloide Lösung vom Niederschlage abgießt und den letzteren mikroskopisch untersucht, so findet man, dałj er kristallinisch ist, obgleich er bei Gegenwart feindisperser Teilchen "amurph" erschien. Das gelang mir z. B. mit "amorphem" Selen durchzufüliren. Auf diese Weise kann mai der Peptisation sich cbenso bedienen, wie des Aufschlämmens zur Trennung eines gröberen Korns vom feinen.

Zum Schlusse dieses Abschnitts führe ich eine Tabelle aller mir aus der Literatur bekannten Fälle der einfachen Peptisation an (siehe nächste Seite).

In den beiden mit ) bezeichneten Fällen sind, soviel ich weiB, die komplexen Verbindungen nicht getrennt worden; jedoch steht ihre

1) Koll.-Zeitschr. 4, 127 (1909). 


\begin{tabular}{|c|c|c|}
\hline $\begin{array}{l}\text { Der zu peptisierende } \\
\text { Niederschlag }\end{array}$ & Der Peptisator & $\begin{array}{c}\text { Die im Dispersionsmittel } \\
\text { leichtiosliche chemisclie } \\
\text { Verbindung }\end{array}$ \\
\hline $\mathrm{Al}_{2} \mathrm{O}_{3} \cdot \times \mathrm{H}_{3} \mathrm{O}$ & Alkalien & Aluminate \\
\hline do. & $\begin{array}{c}\text { Såuren und leich } \\
\text { hydrolisierbare Salze } \\
\text { dieser Säuren }\end{array}$ & $\begin{array}{l}\text { Neutrale und basische } \\
\text { Aluminiumsalze z. } \mathrm{B} \text {. } \\
\qquad \mathrm{AlCl_{3 }}\end{array}$ \\
\hline $\begin{array}{l}\mathrm{Fe}_{2} \mathrm{O}_{3} \cdot \times \mathrm{H}_{2} \mathrm{O} \text { und } \\
\text { ihm ahnliche Hydro- } \\
\text { xyde u. Hydroxydule }\end{array}$ & \multicolumn{2}{|c|}{ Erscheinungen anlog denen des Al-Hydroxyds } \\
\hline $\mathrm{SiO}_{2} \times \mathrm{H}_{2} \mathrm{O}$ & Alkalien & Silikate \\
\hline do. & Säuren & Komplexe Verbindungen *) \\
\hline Sulfide & Schwefelwasserstoff & Komplexe Verblrdungen *) \\
\hline $\begin{array}{c}\text { Schwerlösliche } \\
\text { Ag-Salze }\end{array}$ & $\begin{array}{c}\text { AgNO } \\
\text { vieler Metalle }\end{array}$ & $\begin{array}{c}\text { Gut bekannte komplexe } \\
\text { Salze }\end{array}$ \\
\hline
\end{tabular}

Existenz, wie ich gleich anführen werde, auBer allem Zweifel: Nach der Ionentheorie erhölıt ein Zusatz von solchen Substanzen zum gelösten Körper, die mit ihm gleiche Jonen besitzen, nur dann seine Löslichkeit, wenn komplexe Verbindungen entstehen. Die Herstellung deutlicher Kristalle von $\mathrm{Si}$-Hydroxyd aus starken salzsauren Lösungen (Doveri) und von Sulfidkristallen bei starker $\mathrm{H}_{2} \mathrm{~S}$-Konzentration in der Lösung (de Sénarmon, C. Doelter u. a.) sind offenbare Beweise für die Löslichkeitserhöhung.

Ich habe schon früher erwännt ${ }^{1)}$, daß die von mir durch die Kondensationsmethode erhaltenen gallertartigen Substanzen sich leicht peptisieren lassen müssen und sie werden auch in der Tat peptisiert. Wie bekannt, habe ich in feindispersem kolloiden Zustande Sulfate. Karbonate, Phosphate $u$, a. erbalten; in vielen Fällen können als Peptisatoren für die Sulfate Schwefelsäure, für Karbonate Kohlensüure ${ }^{2}$ ) usw. dienen, denn es bilden sich bei hohen Konzentrationen dieser Săuren deutlich lösliche saure Salze; als Dispersionsmittel ist ein Gemisch von Wasser mit Alkohol zu empfehien.

1) Koll.-Zeitschr. 5, 68 (1909).

7) Meiner Meinung nach sind die von C. Neuberg und B. Rewald beobachteten Erscheinungen (ct. Koll.-Zeitschr. 2, $321-327,354-357,1908$ ) mit den Karbonaten auf Peptisation zuriickzufithren. 
In Anbetracht dessen, daB A. Lottermoser auf diesem Gebiete schon lange, wie schon früher mitgeteilt "), tätig ist, habe ich keine systematischen Untersuchungen betreffend die Peptisation durch Zusatz von verschiedenen lonen ausgeführt und führe auch augenblicklich solche nicht aus.

Das durch Experimente erlangte Material genügt schon zur Erhărtung meiner Peptisationstheorie; es ist leicht durch meine Methode der Reaktionen in konzentrierten L.osungen einen beliebigen Korrper als einen kolloiden Niederschlag zu erhalten, und es bietet keine Schwierigkeiten, den zugehörigen Peptisator zu finden. Also: durch Peptisation kann jeder Körper in kolloide Lösung übergeführt werden.

\section{Beispiele der zusammengesetzten Peptisation.}

Ich begnũge mich mit zwei Beispielen:

1. Bereiten wir uns einen feindispersen Niederschlag von $\mathrm{BaSO}_{4}$ aui folgende Weise $\left.{ }^{2}\right)$ : Nehmen wir zwei sehr kleine Tröpfchen von gesättigten $\mathrm{Mn} \mathrm{SO}_{4}$ - and $\mathrm{Ba}(\mathrm{CNS})_{2}$-Lösungen und mischen sie zusammerı; die dabei erhaltene Gallerte vermengen wir mit $3 \mathrm{ccm}$ einer kalten (20 C) ca. 0,1 Proz. starken Agar-Agar-Lösung (die Agar-AgarLösung ist schon zwei lage früher bereitet und bei einer Temperatur von $20^{\circ} \mathrm{C}$ in hermetisch geschlossenem Gefä̉ aufbewahrt worden), dann erhalten wir eine weiße durchscheinende gallertartige Masse. Diese Masse übergieBen wir in einem großen Becherglase mit $500 \mathrm{ccm}$ einer 0,1 proz. Agar-Agar-Lösung, rühren um und erwärmen sie. Zu Beginn des Errwärmens sammelt sich im oberen Teile des Glases eine voluminöse halbdurchsichtige Gallerte von milchweißer Opaleszenz, unten dagegen ist die Lösung vollkommen durchsichtig. Bei weiterem Erwärmen und Rühren mit einem Glasstabe zergeht die Gallertschicht und wir erhalten eine opaleszierende Flüssigkeit; so wie sie anfängt zu kochen, unterbricht man das Erhitzen, und die heiße Flüssigkeit wird durch ein doppeltes Filter in ein hermetisch geschlossenes GefäB filtriert; man erhält ein schwach opaleszicrendes Filtrat, das im Laufe der Zeit (in 5 bis 10 Tagen) immer trüber wird, bis schließlich in der Lösung sich gallertartige Flocken (Agar-Agar $+\mathrm{BaSO}_{1}$ ) bilden, deren spezifisches Gewicht sehr nahe dem des Wassers kommt. denn bis jetzt (nach Verlauf von 10 Monaten) befinden sie sich in der Flüssig-

1) Koll,-Zeitschr. 4, 130 (1909).

2) Berichtet der nuss. chem. Ges. 7. (20.) Mai 1909. 
keit noch in der Schwebe und es ist noch kein Niederschlag weder an der Oberflarbe der Flüssigkeit, noch auch am Boden des GefäBes wahrzunehmen. Nimınt man aber größere Mengen $\mathrm{BaSO}_{4}$, so wird durch dasselbe fast alles Agar-Agar in den Niederschlag mitgerissen'). Um mich davon zu überzeugen, habe ich folgenden Versuch angestellt: $100 \mathrm{ccm}$ einer vom Niederschlage $\left(\mathrm{Ba} \mathrm{SO}_{4}+\right.$ Agar-Agar) abgeheberten Lòsung engte ich auf 2 bis $3 \mathrm{ccm}$ en und goB sie in ahsoluten Alkohol; erst nach elmiger Zeit vildete sich eine Trübung von nur in sehr geringem Quantum ausfallendem Agar-Agar. Dieses Experiment zeigt deutlich, das die wahre Löslichkeit von Agar-Agar bei Zimmertemperatur äußerst gering ist und daß die größere Menge desselben sich in suspensoidem Zustande befindet. Es wird also bei starker Konzentration von Agar-Agar im Verhältnis zur Menge des gallertartigen $\mathrm{BaSO}_{4}$-Niederschlages das letztere $\mathrm{pept}$ i s i e $\mathrm{t}$ und ungekehrt $\mathrm{BaSO}_{4}$ pektisiert Agar-Agar. Der eben geschilderte Vorgang ist außerst einfach: die dispersen Teilchen von Agar-Agar und $\mathrm{BaSO}_{4}$ werden durch Kapillarkräfte in zusammengesetzte disperse Teilchen verbunden, deten Eigenschaften von ihrer Zusamunensetzung abhängen ( $\mathbf{x}$ disperse Agar-Agar-Teilchen $+\mathbf{y}$ disperse $\mathrm{BaSO}_{4}$-Teilchen), die wiederum von der relativen $\mathrm{BaSO}_{4}$-Menge und Agar-Agar im Dispersionsmittel abhängt. Wenn im zusammengeset:ten Teilchen viele disperse $\mathrm{BaSO}_{4}$-Teilchen vorhanden sind, so erfolgt die $\mathrm{Pektisation}$, im umgekehrten Falle - die Peptisation. Man sieht, dati der geschilderte Fall der Peptisation schwerlöslicher gallertartiger Niederschläge durchaus allgemein ist.

2. Das zweite Beispiel steht im nahen Zusammenhang mit dem sogenanuten "Schutzkolloid“: Wenn man bei der Darstellung einer kolloiden $\mathrm{CuCl}_{2}$-Lösung in Benzol $^{2}$ ) die Reaktion zwischen einer Lösung von oleinsaurem Kupłer in vollständig entwässertem Benzol einerseits und trockenem Chlorwasserstoff andererseits effolgen läBt, z. B. bei einer Konzentration $\frac{N}{1000}$, so entdeckt man, daB nur bei einem Ueberschuß von oleinsaurem Kupfer stabile kolloido Lösungen von Kupferchlorid erhalten werden; führt man aber die Reaktion zu Ende d. h. in Abwesenheit von oleinsaurem Kupfer, bei einer Kon-

1) Journ. d. russ. chem. Ges. 41, 322, 728 (1909). Koll.-Zeitschr. 6, 179, $180(1910)$.

2) Kupferchlorid habe ich im Jahre 1906 (Koll.-Zeitschr. 2, 304) in kolloidem Zustande hergestellt; jetzt habe ich zusammen mit J. B. Kahan die Darstellungsbedingungen quantitativ bestimmt. (Journ. d. russ. chem. Ges. 42, 372, 1910.) 
zentration $\frac{\mathrm{N}}{1000}$, so scheidet sich ein gallertartiger flockiger $\mathrm{CuCl}_{2}$. Niederschlag aus. Das oleinsaure Kupfer, gelöst in Benzol, stełlf eine echte Lơsung eines hochmolekularen Körpers dar, dessen Moleküle, wenn sie sich de facto auch in gelöstern Zustande befinden, doch von den lösenden Kräften geringer Intensităt, verglichen mit den lösenden Kräften im System $\mathrm{H}_{4} \mathrm{O}+\mathrm{NaCl}$, zusammengehalten werden. Praktisch ist $\mathrm{CuCl}_{2}$ uniosslich in Benzol, deshalb wirken in reinem Benzol auf die Oberfläche der dispersen $\mathrm{CuCl}_{2}$-Teilchen kapillare Kräfte sehr geringer Intensität im Vergleich $z u$ den losenden Kräften, welche die Moleküle des oleinsauren Kupfers im Benzol zusammenhalten.

Hieraus sieht man, daB, ebenso wie im ersten Beispiel, die zusammengesetzten dispersen Teilchen ( $x$ disperse $\mathrm{CuCl}_{2}$-Teilchen $+\mathbf{y}$ oleinsaure Kupferınoleküle) mehr oder weniger die Fähigkeit besitzen, im Benzol in suspensoidem Zustande sich zu halten; die Zusammensetzung der dispersen Teilchen hängt von der jeweiligen Konzentration an $\mathrm{CuCl}_{2}$ und an oleinsaurem Kupfer im Benzol ab. Bei geringer Konzentration der dispersen Teilchen im Benzol und bei einem Ueberschub an oleinsaurem Kupfer erhălt man stabile suspensoide Lösungen; im entgegengesetzten Falle muB das oleinsaure Kupfer von den dispersen $\mathrm{CuCl}_{2}$-Teilchen in den Niederschlag mitgetissen werden. Beides kommt vor; um aber alles oleinsaure Kupfer aus der echten Benzollösung auszuscheiden, ist eine große Menge von dispersen $\mathrm{CuCl}_{2}$-Teilchen erforderlich; durch Experimente ist es festgestellt, daB unter den angeführten Bedingungen ein beträchtliches Quantum von oleinsaurem Kupfer als Niederschlag ausgeschieden wird (man erhält einen grünlichbraunen und nicht einen schwarzbraunen Niederschlag von $\mathrm{CuCl}_{2}$ ).

Aus dem eben ausgeführten Peptisationsprozeß wäre es falsch zu schlubfolgern, daB, wenn im Dispersionsmittel disperse $\mathrm{CuCl}_{2}$-Teilchen und irgendeine in diesem Mittel leichtlösliche Substanz sich befinden, die letztere unbedingt $\mathrm{CuCl}_{2}$ peptisieren müBte; es ist notwendig, daB auch die folgende Bedingung erfüllt wird: eine leichtlosliche Substanz (Peptisator) muB sich dauernd in der Oberflächenschicht der dispersen Teilchen halten infolge ihrer Auflösung in der betreffenden Schicht und der Bildung einer kapillaren Verbindung. Wenn z. B. die oben erwähnte Reaktion bei $\frac{\mathrm{N}}{1000}$ in aquivalenten Verhaltnissen ausgefïhrt 
wird, so erfolgt sofort ein Ausscheiden des $\mathrm{CuCl}_{2}$-Niederschlages, wenn auch in der Lossung die im Benzol leichtlösliche Oleinsäure sich befindet.

\section{Von der Stabilităt suspensoider Lösungen.}

A. Die Fähigkeit der dispersen Teilchen, sich gegenseitig zu veröinden.

Stellen wir uns eine echte muiekulare Lösung itn Dispersionsmittel vor, die durchaus nicht imstande ist, die gelösten Moleküle in lonen zu zerspalten; theoretisch können wir die disperse Phase einer derartigen Lösung durch Kataphorese trennen; mit anderen Worten, wenn wir ins Dispersionsmittel eine elektrische Energie einführen, so verăndern wir die Lösungsfähigkeit desselben und die gelösten Moleküle sammeln sich an einem von den beiden Polen; ging die Kataphorese langsam vor sich, so mut sich die Substanz in deutlichen Kristallen am Pol ausscheiden. Nach Unterbrechung der Kataphorese und nachdem das Dispersionsmittel in den ursprüngliclıen Zustand zurückgekehrt ist, muB die am Pol ausgeschiedeue Substanz wiederum in eine echte Lösung unter dem Einfluß der lösenden Krăfte imı Dispersionsmittel ubergehen.

Nehmen wir jetzt eine suspensoide l.ŏsung und unterwerfen wir dieselbe der Kataphorese, so werden an einem der Pole die suspendierten Teilchen sich sammeln; unterbrechen wir die Kataphorese, so werden dieselben nicht immer imstande sein. von selbst wieder in den suspensoiden Zustand üherzugehen. Der reversible Prozeb hăngt im gegebenen Falle von zwei Faktoren ab, erstens vom Dispersitătsgrad der Teilchen in der suspensoiden Lösung vor der Kataphorese und zweitens von der Beschatrenheit des Dispersionsmittels.

Ich will den Einflub dieser Faktoren sofort näher untersuchen:

1. Nicht nur die einzelnen Moleküle sind in der Lage, bei ihrem gegenseitigen Zusammentreffen $z$ "Ileichartigen Kristallen auszilwachsen, sondern auch hochdisperse Teilchen besitzen diese Fähigkeit. Abhängig vom Dispersitatsgrad wechselt diese Fahigkeit ihre Intensităt und mit letzterer auch den Charakter der Verbindung disperser Teilchen. Bei einem dem molekularen nahen Dispersitâtsgrad verbinden sich die dispersen Teilchen, ähnlich den einzelnen Molekülen zu einem gleichartigen Kristallganzen, aber allerdings langsamer als die Moleküle. Bei weiterer Verringerung des Dispersitătsgrades wachsen dic Teilchen anfangs zu einem Aggregat, das sich schneller oder langsamer z.4 
einem Kristallganzen -- zu einem gieichartigen Kristall - umformt. Die Stabilitat der sic- zuerst bildenden Aggregate ist um so geringer, je größler die diese Aggregate zusammens zenden dispersen Teilchen sind.

Ich mub bemerken, daB auBer der angeführten "Kristallisationsverbindung " der dispersen Teilchen, unter dem Einflub verschiedener Gründe es auch eine "Kapillarverbindung" geben kamn; so dienen Stäubchen in der $\mathrm{BaSO}_{4}$-Susfension so\%usagen als Zentra der Kondensation für die dispersen Teilchen $\left.{ }^{1}\right)$. Die „Kristallisationsverbindung" der Teilchen ist stabiler als die "Kapillarverbindung", und deshalb muB man besonders die erstere vermeiden, falls man Suspensoide reversibel crhalten will. In einigen Fallen ist es zwischen „Kristallisations-" und „Kapilliır"Verbindungen unmöglich, einen scharfen Unterschied durchzuíühren.

2. Die Beschaffenheit des Dispersionsmittels ist auch von wesentlichem EinfluB; ein Beispiel wird das erläutern: Befand sich eine suspensoide Lösung im Zustande der einfachen Peptisation, so geht nach Unterbrechung der Kataphorese sogar ein verhaltnismäBig grobdisperser Niederschlag von neuern dank der Peptisation in Lösung über; fehlen aber die Bedingungen der Peptisation, so ist die Reversibilität des Prozesses wenn auch möglich, so doch in seltenen Fallen tatsächlich nur dann, wenn die feindispersen Teilchen keine stabilen grobdispersen Aggregate dank der " $\mathrm{Kr}$ is $\mathrm{t}$ a ll i sa ti on s ve r b in d ung " disperser Teilchen ergaben; solche Erscheinungen beobachtet man bei den suspensoiden Lösumgen hochmolekularer Körper, wie z. B..bei der suspensoiden Agar-Agar-Lösung, welche bei Zimmertemperatur nicht gelatiniert.

\section{B. Peptisation und Stabilität suspensoider Lösungen.}

1. Die Möglichkeit, eine suspensoide Lösung gerịger Konzentration in einem, in bezug auf die disperse Phase vollständig passiven Dispersionsmittel herzustellen.

Stellen wir uns ein in bezug auf irgendeine disperse Substanz chemisch und physikalisch passives Dispersionsmittel $x$ vor, mit anderen Worten, nehmen wir an, der Körper $x$ löst sich absolut nicht, weder physikalisch noch chemisch im Dispersionsmittel: die Substanz $x$ ist in verschiedenen Dispersitătsgraden gegeben, z. B. von $200 \mu \mu$ bis $2 \mu \mu$. Es unterliegt keinem Zweifel, daB man eine Suspension ge-

1) Koll.-Zeitschr, 3, Taf. IX, Fig. 4 (1908). 
ringer Konzentration im passiven Dispersionsnitiel herstellen kann, denn es liegt im Bereich der Möglichkeit, in das Dispersionsmittel disperse Teilchen von so kleinen Dimensionen und in so geringer Anzahl einzuführen, daß die Entfernungen der Teilchen voneinander sehr bedcutende sind und infolgedessen der ProzeB der Bildung des Niederschlages und $\mathrm{d}+\mathrm{r}$ gegenseitigen Verbindung beträchtlich ausgedehnt wird. Die rage der Herstellung einer suspensoiden Lösung geringer Konzentration in passiven Dispersionsmittel ist nicht so einfach. Man könnte glauben, dak The Svedbergb diese Frage in negativem sime entscheidet, was aber meiner Meinung nach nicht in seiner Absicht liegt, denn das Nichterhaltes von Pt-Sol im reinsten $\mathrm{C}_{2} \mathrm{H}_{5}, \mathrm{O}, \mathrm{C}_{2} \mathrm{H}_{3}$ durch elektrische Zerstäubung von metallischem $P_{t}$ spricht doch nur dafür, dab man ein Pt-Sol unter den obigen Verhältnissen nicht durch elektrische Zerstäubung erhalten kamn. leh bin der Alssicht, daß es durchaus möglich ist, ein Pt-Sol von geringer Konzentration in Aether durch Kondensation der PtMoleküle (z. B. sehr verdünnter Pr-1)ämpfe) im genannten Dispersionsmittel zu erhalten. In der Tat geht aus der Formela ${ }^{2}$ )

$$
W=K \cdot Q-L
$$

klar hervor, daf man bei genügend kleinem $Q-L$ und sogat bei verschwindend kleinem $L$ die Kondensation heliebig verlangsamen kann; wir werden also im Laufe der Kondensation ene suspensoide Lösung haben. Einem jeden wird es einleuchten, dats dic cinzelnen vonein-

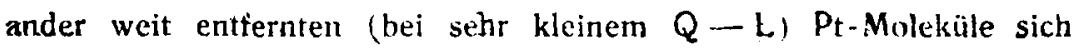
nicht bald zu schnell zu Boden sinkenden Komplexen knndensieren werden.

Die Passivitä des Dispersionsmittels ist also, wie aus meiner Peptisationstheririe hervorgeht, auf die Stabilität suspensoider Losungen in dem Sinne von Einfluß, dal sie nicht dle Darstellung stabiler Sole hoher Koizzentration gestattet, jedoch nicht vollständig die Möglichkeit ausschliehit, stabile Sole von genügend geringer Konzentration zu erhalten.

Wie ich. schon in einer früheren Abhandlung ${ }^{3}$ ) erwähnt habe, werden die dem molekularen Dispersitätsgrad nahen und ini passiven Dispersionsinittel befindlichen dispersen Teilchen durch den inneren Druck des Dispersionsnittels sozusagen einander entgegengestoßen,

1) Koll.-Zeitschr. 2, 146 (1907).

2) Koll.-Zeitschr. 6, 210 --213.(1910),

s) Uebetsättigung und Unterkuhlung usw. Kolloidehem. Beih. 1, 338(1910). 
denn man kann im angeführten Falle nicht wie für grơBere in. eine Flüssigkeit eingetauchte. Körper annehmen. dab der innere Druck keine Wirkung ausübt. Wenn wir zwischen die Moleküle eines physikalisch und chemisch vollständig passiven Dispersionsmittels allerfeinste disperse Teilchen einführen, so müssen wir in der Tat eine Arbeit gegen die gegenseitige Anziehung der Moleküle des Dispersionsmittels, mit anderen Worten, gegen den Normaldruck auf die Oberflăche des Dispersionsmittels verrichten. Daraus folgt, dab das Dispersionsmittel um so mehr die Kondensation der in dasselbe gewaltsam einge ührten feindispersen Teilchen in grobdisperse Aggregate begünstigt, je größer der Normaldruck ist.

\begin{tabular}{c|c|c}
\hline & $\begin{array}{c}\text { Normaldruck in Atm. } \\
\text { hei 200 }\end{array}$ & $\begin{array}{c}\text { Dielektrizitatskonstante } \\
{[\lambda==\infty ; t=18]}\end{array}$ \\
\hline $\mathrm{H}_{2} \mathrm{O}$ & 18000 & 81 \\
$\mathrm{C}_{2} \mathrm{H}_{5} \cdot \mathrm{OH}$ & 3600 & 27 \\
$\mathrm{C}_{2} \mathrm{H}_{5} . \mathrm{O} \cdot \mathrm{C}_{2} \mathrm{H}_{5}$ & 1600 & 4
\end{tabular}

Diese Tabelle der Normaldrucke und Dielektrizitätskonstanten einiger Flüssigkeiten habe ich angeführt, damit nicht aus dem oben angeführten Einflusse des Normaldruckes auf die Stabilitär kolloider Lösungen irrige Schlubfolgerungen gezogen werden. Aus dieser Tabelie ersieht man, daB, wenn man für die drei bezeichneten Flüssigkeiten im Verhaltnis zu ihnen eine vollkommen gleich passive disperse Phase findet, man fürs Wasser bei derselben Konzentration der dispersen Phase in allen drei Mitteln eine kolloide Lösung geringster Stabilităt erhält. Andererseits zeigt die Tabelle, daß) es äußerst schwierig ist, eine für das Wasser gleich passive Phase wie für Aether zu finden, denn die Reaktionsfähigkeit des Wassers $(\Gamma \neq 81)$ ist bedeutend gröller als beim Aether $(D=4)$. Es ist deshalb auch im Wasser leichter als im Aether, eine verhältnismäBig konzentrierte suspensoide $\mathrm{P}_{\mathrm{t}}$-Lösung $\mathrm{zu}$ erhalten. Ich mub hinzufügen, dath, genau genoinmen, der Einflub des Normaldruckes auf den Kondensationsproze $B$ und die Stabilität kolloider Losungen nur für Körper gilt, die fast absolut unlöslich sind und sich passiv zum Dispersionsmittel verhalten.

2. Die Peptisalionsbedingungen als direkte Faktoren der Stabilitat disperser Systeme.

Aus allem Angeïulırten geht klar hervor, daB die. Peptisations$b$ edingungen in dispersen System von großem Einfluß auf die Stabilität 
sind. Es ist in der Tat nicht schwer zu begreifen, daB, welcher Art auch die von uns zur Trennung der dispersen Phase vom Dispersionsmittel angewandte Energie sei, eine um so grðßere Arbeit geleistet werden mub, je größer die Intensităt der lösenden oder kapillaren (dispergierenden) Krafte an der Berührungsstelle des Dispersionsmitteis mit der Oberflächenschicht der dispersen Teilchen ist. Man darf niemals aus dem Auge lassen, dat das Dispersionsmittel sich in der Oberflächenschicht der dispersen Teilchen löst, ebenso können auch ins Dispersionsmittel gebrachte Fremdkorper sich in derselben losen. In direkter Abhängigkeit von der Pestigkeit, mit der das Dispersionsmittel in Lossung in der Oberflächenschicht der dispersen Teilchen gehalten wird, befindet sich auch die Stabilitat des dispersen Systems.

In meiner Abhandlung ,Zur Lehre von den Zuständen der Materie "') habe ich genügend Beispiele für den stabilisierenden Einfluß der Peptisationsbedingungen angeführt; so habe ich $z$. B. darauf hingewiesen, daB die in einige starke Salzlbsungen gegossenen kolloiden Silberiosungen bei Gegenwart des Luftsauerstoffs nicht koagulieren, sondern langsum peptisiert werden bis zur völligen Auflösung; hei Mangel an Sauerstoff tritt dagegen Koagulation ein

Zum Schluß dieses Abschnitts führe ich einen Versuch an, den ich gemeinsam mit B.W. Malyschew mit Te angestellt habe, um meine Peptisationstheorie nochmals zu kontrollieren. Fs wurden zwei Gefabe A und B mit je $1000 \mathrm{ccm}$ Wasser gefüllt; in das GefäB A wurde durch andsuerndes Kochen moglichst von Luft befreites Wasser gegossen und derartıg hermetisch geschlossen, daß äuBerst wenig Luft über der Oberilkche blieb, in das GefäB B dagegen mit Luft gesättigtes Wasser mit viel Luft über dem letzteren. In beiden Gefälen wurdeı ziemlich grobdisperse Systeme von $T e$ durch schnelles Eingieben von je $5 \mathrm{ccm}$ einer heiben Lösung von $\mathrm{Te}$ in - bei Zimmertemperatur gesättigte - Kalilauge hergestellt $(5 \mathrm{ccm}$ der Lösung enthielten $0,1 \mathrm{~g}$ metallisches $\mathrm{Te}$ ). Das GefäB A wurde sofort mit einem fast die Flüssigkeit berührenden Kork geschlossen, während in das GefäB B einige Minuten hindurch reiner Sauerstoff eingeleitet und die Lossung häufig durchgeschüttelt wurde, um sie mit der darüber befindlichen Luft in innige Berührung zu bringen. wobei die Luft durch häufiges Entkorken erneuert wurde. Die hierbei erhaltenen Resultate fasse ich in folgender Tabelle zusammen:

1) Koll.-Zeitschr. 4, 123-130, 315 $\ldots 317 ; 5,62-68(19(9)$. Ich füge linzu, daB durch Peptisation erhaltene Kolloidlosungen bisweilen den Kolloidlosungen hochmolekularer Körper (wie z. B. Agar-Agar) nahe kommen. 


\begin{tabular}{|c|c|c|c|c|}
\hline & 15 Min. & $\begin{array}{c}12 \text { Std. } \\
\text { Zusammen }\end{array}$ & $\begin{array}{c}48 \text { Std. } \\
\text { gie Ben der Lö }\end{array}$ & $\begin{array}{l}72 \text { Std. } \\
\text { sungen }\end{array}$ \\
\hline $\begin{array}{c}\text { Lösung } \\
\text { AA }\end{array}$ & $\begin{array}{c}\text { eine } \\
\text { schwärzlich- } \\
\text { graue } \\
\text { das Licht nicht } \\
\text { durchlassende } \\
\text { Suspension }\end{array}$ & $\begin{array}{c}\text { geringer } \\
\text { schwarzer } \\
\text { Niederschlag }\end{array}$ & \begin{tabular}{|c|} 
der \\
Niederschlag \\
stärker \\
geworden; \\
die Lösung \\
über demselben \\
fast lar:
\end{tabular} & dasselbe \\
\hline $\begin{array}{c}\text { Lösung } \\
\text { B }\end{array}$ & $\begin{array}{c}\text { eine } \\
\text { schwäralich- } \\
\text { graue } \\
\text { das Licht nicht } \\
\text { durchlassende } \\
\text { Suspension }\end{array}$ & $\begin{array}{l}\text { schwarzgraue } \\
\text { das Licht gut } \\
\text { durchlassende } \\
\text { suspensoide } \\
\text { Lösung }\end{array}$ & $\begin{array}{l}\text { hellgraue } \\
\text { suspensoide } \\
\text { Lösung }\end{array}$ & $\begin{array}{c}\text { farblose } \\
\text { schwach ge- } \\
\text { trübtelösung; } \\
\text { der Ueber- } \\
\text { gangsprozeB } \\
\text { des Te in tellur- } \\
\text { saures Kali } \\
\text { ist fast beendet }\end{array}$ \\
\hline
\end{tabular}

Nach 72 Stunden wurde das Gefäß A geöffnet und durch häufiges Schütteln mit der Luft in Berührung gebracht; nach 240 Stunden erhielt man eine hellgraue Kolloidlösung.

Dieses Beispiel, nicht weniger anschaulich als das früher angeführte mit Silber, bewrist die Richtigkeit meiner Theorie.

\section{Die Analogie und der Unterschied zwischen echten und kolloiden l.osungen $\left.\left.{ }^{1}\right)^{2}\right)$.}

1. Der gasförmige una der gelöste Zustána.

Wie bekannt, ist für Gase von geringer räumlicher Konzentration (resp. Dichte) die folgende Gleichung anwendbar:

$$
\mathrm{pv}=\mathrm{RT}
$$

für Gase von bedeutender räumlicher Konzentration (resp. für Gase unter hohem Druck) dagegen muß man schon sowoll mit der Raumausdehnumg der Molekïle als auch mit den Attraktionskräften rechnen. Die Ausdehnung der Moleküle verkleinert natürlicherweise

1) Vgl. Koll.: Zeitschr. 2, 202-205 (1908); 5, 119 (1909).

2) Dieser Absclınitt ist zuerst in Nr. 5 der ,Berichte des Berginstituts* 1910 abgedruckt worden. 
den Inhalt des Raumes, in dem die molckulare Bewegung vor sich geht, infolgedessen wird die Häufigkeit des molekularen Anpralls (resp der Druck) größer als bei Molekülen, die nicht über eine derartige Ausdehnung verfügen. Das Vorhandensein der Aftraktionskräfte dokumentiert sich durch Veränderung der molekularen Trajektorie; infolge der Attraktion sind die einzelnen Abschnitte der molekularen Trajektorie nicht mehr gerade, sondern gekrümmte Linien, wobei die Krümmung um so größser ist, je intensiver die molekulare Attraktion. Die molekularen Attraktionskräfte beeinflussen den vom Gas nach auken ausgeübten Druck, denı die Kraft der molekularen StöBe verringert sich infolge der gegenseitigen Auziehung der Moleküle. Es folgt daraus, dab die Gileichung (I) für Gase von bedeutender räumlicher Konzentration nur dann anwendbar sein wird, wenn man in geeigneter Weise den Druck und das Volum modifiziert. Eine in diesem Sinne modifizierte Gleichung wird, wie einem jeden tekannt sein dürte, "die van der Waals'sche Zustandsgleichung" genannt und kann 7. B. folgendermaßen zum Ausdruck gebraclit werden:

$$
\left(p+K_{1}\right)\left(v+K_{i j}\right)=R T
$$

Es wird allgemem für de" gasförmigen Zustand angenommen, daß das Dispersionsmittel, d. h. "Leere", von keinem Einfluß auf die Bewegung der Moleküle's ist; zieht inan aber die Anziehung zwischen der "Leere" und den Gasmolekülen in Betracht, so müßte die Gleichung (II) folgende Form annetmen:

$$
\left(p+K_{1}+K_{2}\right)\left(v+K_{3}\right)=R \cdot T \text {. }
$$

Jetzt möchte ich zeigen, dab die Gleichung (III) für den gelösten $\mathrm{Zu}$ stand im allgemeinen anwendbar ist. In echten Lösungen bedeutender Konzentrationen ist von Bedcutung nicht nur die gegenseitige Anziehung der gelösten Moleküle, sondern nuch die Anzichung zwischen! den Molekülen des gelösten Körpers und dem Dispersionsmittel, und die lezztere ist von Einfluk auf die Krümmung der Trajektorien der gelösten Moleküle, und zwar uni so mehr. je größer die Intemsităt der Anzielung ist. Auf Grund des Angeführten wird die Gieichung für den gelosten Zustand aiso lauten:

$$
\left(\mathrm{P}+\mathrm{K}_{1}{ }^{\prime}+\mathrm{K}_{2}{ }^{\prime}\right)\left(\mathrm{V}+\mathrm{K}_{\mathbf{}^{\prime}}{ }^{\prime}\right)=\mathrm{R} \mathrm{T}
$$

worin $P$ den durch ein Osmometer gemessenen osmotischen Druck bedeutet. Der Faktor $K_{1}^{\prime}$ ist ebenso wie in der va $n$ der Wa als'schen Gleichung positiv, $K_{3}{ }^{\prime}$ dagegen negativ; es ist nicht schwer nachzu-

1) Vgl. meine Anschaunng von , Leere". Koll.-Zeitschr 2, 270 (1908); 5, 157 (1909). 
weisen, daß $K_{2}{ }^{\circ}<0-$ mit anderen Worten, dank dem Vorhandensein von Attraktionskräften zwischen den Molekülen des Lösungsmittels und des gelösten Körpers ist der beobachtete osmotische Druck größer als unter denselben Bedingungen bei Gasen. In der Tat befindet sich im Osmometer die Lösung, außerhalb der halbdurchlesssigen Membran das Lösungsmittel; das letztere wird in das Osmometer nicht nur durch die molckularen Stöße der gelösten Moleküle an die Membran hineingetrieben, sondern infolge der unnittelbaren Anziehung durch diese Moleküle der Moleküle des Lösungsmittels. Es ist selbstverständlich, daB von einer Verringerung der Stobkraft der gelösten Moleküle hierbei nicht die Rede sein kann, denn im gegebenen Falle gibt es nicht in dem Sinne wie bei Gasen eine Oberflächenschicht; im Gegenteil, das Vorhandensein von Lösungsmittel auf der einen Seite der Membran, dessen Moleküle die gelosten Moleküle an sich zu ziehen bestrebt sind, wird die Stärke des StoBes erhöhen. Indem man also sich an die allgemein angenommenen!) Ansichten über den gasförmigen Zustand hält, ıußB man zum Schlusse gelangen, daß der gelöste Zustand sich wesentlich vom gasförmigen durch das Vorhandensein der Attraktion zwischenden gelösten Molekülen und den Molekülen des Dispersionsmittels unterscheidet, was durch die Krünmung der molekularen Trajektorie und durch Erhöhung des osmotischen Druckes im Osmometer um eine gewisse Grobe im Verhältnis zum Gas zum Ausdruck gelangt.

Es ist nicht schwer zu begreifen, dab diese Erhöhung auch für jente verdünnten Lösungen, welche keine besondere Anziehung zwischen den Molekülen des Lösungsmittels und des gelösten Körpers offenharen, nicht grob ist und man kann annehmen:

$$
\text { P. } V=R T \text {. }
$$

Ich werde inich hier nichi mit der Erklärung der Frage vom geringfügigen Einfluß der Atraktionskräfte auf den osmotischen Druck befassen, sondern beschränke mich darauf, ein Zitat aus emer meiner früheren Abhandlungen anzufüliren ${ }^{2}$ ): "Die Bindung des Wassers in den Kristalloidhydraten sinkı mit Verminderung der Gröbe des krıstälinen Kornes. Anfangs zeigt sich das in der erhöhten Spannung der Dämpfe und in Sinken der Uebergangspunkte in niedere Hydrate, dann aber in jenen Erscheinungen, denen der Ausdruck ,Adsorptions-

1) Vgl. die vorhergehende Anmerkung.

z) Koll.-Zeitschr. 2, 374 (1908). 
wasser' entsprungen ist. $\mathrm{Da}$ in Lösungen keine anderen kristallinen Massen, ats nur solche von äußerster Kleinheit vorkommen können, so kann auch das Kristailwasser beim Uebergang des Kristalloidhydrats in Losung sich nicht bemerkbar (in einigen Fullen innerhalb der Grenzen unserer jetzigen Methoden) von der übrigen Wassermenge der Lðsung unterscheiden. Jedoch liegt darin kein Grund, das Vorhandensein von Hydraten in Lösungen zu bestreiten."

2. Der osmotische Druck kolloider Lösungen.

Folgende drei Faktoren sind von Einflub auf den osmotischen Druck der Suspensoide und Emulsoide:

I. Der Dispersitatsgrad.

II. Die Konzentration der dispersen Phase im Dispersionsmittel.

III. Die Attraktion zwischen der dispersen Phase und dem Dispersionsmittel'y.

Für ideale verdünnte molekulardisperse kolloide Lösungen, die physikalisch und chemisch vollkommen passiv in bezug auf die disperse Phase des Dispersionsmittels sind, gilt folgende Zustandsgleichung: P.V $=\mathrm{RT}$.

rur gewonnliche reale kolloide Lösungen haben wir

a) bei Vorhandensein verhältnismäßig bedeutender Attraktion zwischen der dispersen Phase und dem Dispersionsmittel die Gleichung:

$$
\left(\mathrm{P}+\mathrm{K}_{2}\right)\left(\mathrm{V}+\mathrm{K}_{3}\right)=\mathrm{R} \mathrm{T}
$$

b) dagegen beim Fehlen der Attraktion: $P .\left(V+K_{3}\right)=R T$

c) und im allgemeinen: $\left(P+K_{1}+K_{2}\right)\left(V+K_{3}\right)=R T$, wobei natürlich die dispersen Teilchen als Moleküle angesehen werden.

Man kann ein der idealen verdünnten molekulardispersen Kolloidlösung nahe kommendes disperses System bei Verwirklichung der Reaktion der Synthese $A+B=A B$ in absolut nicht lösendem und mit dem Körper $A B$ chemisch keine Reaktion eingehendem Dispersionsmittel erinalten. Damit durch den Kondensationsprozeb, der im erwähnten Fall unvermeidlich vor sich geht, nicht plötzlich viele Teilchen von nicht molekularer Dispersität entstehen, muß man die Reaktion zur Darstellung des Körpers $\mathrm{AB}$ in äußerst verdünnten Lösungen vornehmen; trotzdem kann man nicht verlindern, daß nichtmolekulardisperse Teilchen entstelien, aber im Grenzzustande, d. h. im idealen Fall, kann man annehmen, daB P.V $=\mathrm{RT}$, wobei

3) Besonders bei peptisierten Kolloidlösungen darf man diesen letzten Faktor nicht aus den Auge lassen. 
$\mathbf{P}$ gleich ist dem Gasdruck unter gleichen Bedingungen. Für Kolloidlösungen von geringerem Dispersitătsgrade, die aber physikalisch und chemisch gleich passiv zum Dispersionsmittel sich verhalten, wcrden wir a) bei sehr geringer Konzentration die Gleichung P. V $=R T$ haben, wobei $P$ gleich ist dem Gasdruck bei gleichen Bedingungen (natūrlich wird mit Verkleinerung des Dispersitätsgrades auch $P$ kleiner? und $\beta$ ) bei hoher Konzentration: $\mathrm{P} .\left(\mathrm{V}+\mathrm{K}_{3}\right)=\mathrm{RT}$.

Für peptisierte Kolloidlösungen bekommt die Gleichung in Anbetracht der Attraktion zwischen der dispersen Phase und dem Dispersionsmittel folgende Fassung:

$$
\text { a) } \left.\left(\mathrm{P}+\mathrm{K}_{2}\right) \cdot \mathrm{V}=\mathrm{RT} \quad \text { 穴 }\right)\left(\mathrm{P}+\mathrm{K}_{2}\right)\left(\mathrm{V}+\mathrm{K}_{3}\right)=\mathrm{RT}
$$

Die allgemeine Gleichung $\left(P+K_{1}+K_{2}\right)\left(V+K_{4}\right)=R T$ ist fast ganz ohne Bedeutung für die Kolloidlösungen, weil entweder das Zusammentreffen der dispersen Teilchen selten stattfindet, oder weil in der Mehrzilul der Fälle bei hoher Konzentration und bei hohem Dispersitătsgrad diese Teilchen sich schnell vereinigen, deshalb kann man $K_{1}=O$ annehmen.

Nach den Arbeiten von J. Perrin und The Svedberg zu urteilen, können die Kolloidlösungen als echte Lösungen angenommen werden, wobei als Moleküle die dispersen Teilchen anzusehen sind, ich will aber auf einen wesentlichen, aus folgendem Beispiel zu ersehenden Unterschied zwischen den echten und kolloiden Lösungen hinweisen.

3. Der Unterschied $z$ wischen suspensoiden (kolloiden) und suspensiden (echten) Losungen.

Bereiten wir uns eine ideale verdünnte molekulardisperse suspensoide Lösung eines beliebigen Körpers, so haben wir dafür die Gleichung: $P . V=R T$, dagegen für eine echte Lösung desselben Körpers bei derselben Konzentration im allgemeinen die Gleichung: $\left(p+K_{2}\right) \cdot V=R T$, wobei $p>P$ ist; haben wir ein geeignetes Lösungsmittel gefunden, so können wir uns bis zu einem gewissen Grade $p=P$ nähern, wenn $K_{2}$ sich $O$ nähert, aber wir sind nicht imstande den langsam, jedoch u n verme id $l$ i ch fortschreitenden Kondensationsprozeb, d. h. die Verringerung des Dispersitatsgrades, aufzuhalten. Darin besteht auch der Unterschied zwischen den suspensoiden und suspensiden (echten) Lösungen. Dieser Unterschied wird verringert durch

1) Wie bekannt, ist das Molekulargewicht (die Teilchengrobe) mit dem osmotischen Druck durch die Gleichung $M=22,42(1+0,00367 t) \stackrel{C}{P}$ verbunden, worin $\mathrm{C}$ die Gramm-Anzahl der Substanz im Liter ausdrilckt. 
das Vorhandensein ïbersāttigter Lösungen '). Wenn nun auch vom kinetisch-molekularen Standpunkt aus kein wesent. licher Unterschied $z$ wischen den kolloiden und echten Lösungen eines und desselben Körpers zu konstatieren ist, so existiertdoch ein solchervom Standpunktder Phasen. lehre aus.

Es war bis jetzt die Rede von kolloiden und echien Lösungen eines und desselben Körpers; es versteht sịch nun von selbst, daB, wenn wir echte und kolloide Lösungen verschiedener Körper miteinander vergleichen, es vo n Standpunkte der Phasenlehre aus keinen wesentlichen Unterschiei gibt; der Uebergang ist etwa folgender:

Nicht peptisierte Kolloidlösungen

Peptisierte Kolloidlösungen

Echte Lösungen hochmolekularer Körper (oder Solutoide)

Echte Lösungen nicht hochmolekularer Körper

Echte Lösungen 'der Elektrolyten

Zum Schlusse muß ich bemerken, daß es Ansichten von osmotischen Erscheinungen der Kolloidlösungen gibt $^{2}$ ), die direkt den von mir ausgeführten entgegengesetzt sind; so schreibt A. A. Jakowkin: „Den kolloiden Lösungen, welche einen höchst geringen osmotischen Druck zeigen, schreiben wir gewöhnlich ein sehr großes Molekulargewicht zu. Aber schwerlich kann das in allen Fällen und vollkommen richtig sein, da die kolloiden Lösungen sich auch dadurch auszeichnen, daß sie eine sehr geringe Affinität zwischen dem gelüsten Stoffe und dem Lösungsmittel zeigen. Infolgedessen hängt hier der geringe osmotische Druek nicht nur von dem hohen Molekulargewicht $a b$, sondern auch von der geringen Affinität. Bei den großien Fortschritten der Lehre von kolloiden Lösungen in der letzten Zeit, können wir erwarten, daß man auch Lösungen solcher Kolloide erhalten wird, für welche kein Grund vorhanden sein wird, ein hohes Molekulargewicht anzunehmen."

Ich kann mich mit den Ansichten von. A. A. Jakowk in uicht einverstanden erklären, denn die hochmolekularen Kolloidlösungen

1) Vg1. Koll.-Zeitschr. 5, 212--221 (1909). Ausführlicher uber die Uebergangserscheinungen in "Kolloidwissenschaft und Struktur der Materie" (in Vorbereltung).

2) Zeitschr. f. phys. Chem. 70, 175 - 176 (1910). 
besitzen einen durchaus meBbaren osmotischen Druck, der nicht geringer sein muk als der des Gases unter gleichen Bedingungen. Das Fehlen der "Affinitat" ruft, wie oben gezeigt wurde, entweder eine Kondensation der Moleküle in grobdisperse Teilchen hervor, oder macht den osmotischen Druck dem des Gases gleich, aber auf keinen Fall kleiner.

\section{SchluB.}

In vorliegender Abliandlung habe ich mit Absicht die elektrische Teilchenladung der dispersen Phase und die lonenreaktion nicht erwăhnt, weil ich die .Loslichkeitsbeeinflussung "1) für ein allgemeineres Prinzip halte, als die "Ionenreaktion", die natürlicherweise nur für Elektrolyte in Betracht kommt. Ich zweifle nicht im geringsten daran, daß die Loslichkeit im letzten Falle eng mit der lonenreaktion verbunden ist, denn das geht genügend klar aus den bemerkenswerten Arbeiten von A. Lottermoser hervor. Indessen auch hier scheint mir die Erklärung der Ersrheinungen vom Standpunkt der "Löslichkeitsbeeinflussung viel natürlicher. Ebenso bin ich von der elektrischen Ladung der dispersen Teilchen überzeugt und daB von derselben die Stabilităt kolloider Lösungen abhăngt. Wenn ich aber trotzdem die "Löslichkeitsheeinflussung* voranstelle, so tue ich es aus dem Grunde, weil ich, von dieser Theorie ausgehend, bei meinen Experimenten keine MiBerfolge zu verzeichnen gehabt habe. An der Hand dieser Experimente nun mubte ich zur Ueberzeugung gelangen, daß die Verănderung der lösenden oder kapillaren Krâfte an der Grenzflache zwischen der dispersen Phase und dem Dispersionsmittel, die natürlich von elektrischen und anderen Erscheinungen begleitet wird, vonallergröbtem Einfluß a uf die Stabilität der dispersen Systeme ist. Aus diesem Grunde könnte man die Kolloidchemie und -physik die Chemie und Physik des Oberflächenzustandes der festen und flüssigen Körper bezeichnen.

(Zweite Abhandlung folgt in einem der năchsten Hefte.)

1) Die Bedeutung dieses Prinzips kommt noch deutlicher in ,Kolloldwissenschaft und Struktur der Materie* (in Vorbereitung) zum Ausdruck. 